\section{CT Angiographic Source Images with Modern Multisection CT Scanners: Appropriate Injection Protocol Is Crucial}

I have read with great interest the recently published article of Sharma et al. ${ }^{1}$ The study compared CT angiographic source imaging (CTASI) performed by a modern multisection CT scanner with cerebral blood flow (CBF) and cerebral blood volume (CBV) maps derived from dynamic CT perfusion in patients with acute ischemic stroke. The results demonstrate the strong correlation between the lesion volume of CTA-SI and CBF $(r=0.89, P<.001)$ in contrast to the weak correlation between the lesion volume of CTA-SI and CBV $(r=$ $0.5, P<.001)$. The authors concluded that CTA-SI is CBF- rather than CBV-weighted.

Although it was probably not the intention of the authors, this conclusion suggests that CTA source data with modern multisection CT scanners somehow include CBF information. CBF can be calculated only from dynamic CT data acquired during the first pass of a contrast bolus. Hence, I consider the conclusion of Sharma et $\mathrm{al}^{1}$ critical, and it is the opposite of those of previous reports of the blood volume basis of CTA-SI. ${ }^{2}$ In my opinion, the reported results of apparent CBF-weighted CTA-SI in the article of Sharma et al need further discussion and detailed consideration of the underlying principle of CTA-SI.

CBV relates to the area under the curve and is expressed by the following equation:

$$
\text { 1) } C B V=\frac{\int_{0}^{\infty} c(t) \mathrm{d} t}{\int_{0}^{\infty} v(t) \mathrm{d} t} \quad\left[\mathrm{~mL} \cdot 100 \mathrm{~g}^{-1}\right] \text {, }
$$

where $c(t)$ is the tissue concentration and $v(t)$ is the vascular concentration of the marker at certain time points $(t)$. For CT, this is only fulfilled with a dynamic CT data acquisition. As cited by the authors, CTA-SI, like the related techniques of perfusion-weighted CT and perfused blood volume (PBV) imaging, is based on the principle that was originally reported by Hamberg et al. ${ }^{2}$ With the assumption of vascular and tissue contrast steady-state, the above calculation of CBV can be reduced to the following equation:

$$
\text { 2) } C B V=\frac{\max c(t)}{\max v(t)} \quad\left[\mathrm{mL} \cdot 100 \mathrm{~g}^{-1}\right]
$$

where $\max c(t)$ is the maximum tissue concentration and $\max v(t)$ is the maximum vascular concentration of the marker. This consideration eliminates the need for a dynamic CT data acquisition and is the basis of CTA-SI. Blood volume information can be extracted from CTA data when the data acquisition is performed at the plateau phase of contrast injection with vascular and tissue-contrast steady-state.
With former generations of CT scanners, dedicated injection protocols in CTA-SI were not that crucial because the slow scanning time "automatically" resulted in appropriate bolus configuration to fulfill the algorithm of CTA-SI. In contrast, the fast scanning times with modern CT scanners require very accurate bolus timing for CTA-SI. Sharma et $\mathrm{al}^{1}$ used an injection protocol with a delay between 5 and 10 seconds, up to $90 \mathrm{~mL}$ of $300-\mathrm{mg}$ iodine $/ \mathrm{mL}$ concentrated contrast agent, a flow rate of $5 \mathrm{~mL} / \mathrm{s}$, and no saline flush. With the fast scanning time of the 64-section CT scanner used, this injection protocol results in a distinct arterial contrast. However, the mandatory assumption for CTA-SI with vascular and tissue contrast steady-state according to the considerations of Hamberg et $\mathrm{al}^{2}$ is thereby violated. Hence, the size of the CTA-SI lesion is overestimated.

It is not surprising that Sharma et $\mathrm{al}^{1}$ found a better correlation between CTA-SI with CBF, a parameter estimating infarct core and penumbra, and not with $\mathrm{CBV}$, a parameter reflecting the infarct core. However, this result does not imply causality but is the consequence of the injection protocol used. It has been recently demonstrated that using an injection protocol with individualized delay for peak enhancement in the superior sagittal sinus, $80 \mathrm{~mL}$ of $370-\mathrm{mg}$ iodine $/ \mathrm{mL}$ concentrated contrast agent, a flow rate of $4 \mathrm{~mL} / \mathrm{s}$, and $50 \mathrm{~mL}$ of saline flush resulted in a strong correlation between the lesion volume of PBV with CBV $(r=0.922, P<.01)$ when performed by a modern multisection CT scanner. ${ }^{3}$ In consequence, the results of Sharma et al do not contradict the recommendations of the American Heart Association for CTA-SI ${ }^{4}$ but point out the importance of appropriate injection protocol in conjunction with modern multisection CT scanners to fulfill the requirements for the appropriate use of CTA-SI.

\section{References}

1. Sharma M, Fox AJ, Symons S, et al. CT angiographic source images: flow- or volume-weighted? AJNR Am J Neuroradiol 2011;32:359-64

2. Hamberg LM, Hunter GJ, Kierstead D, et al. Measurement of cerebral blood volume with subtraction three-dimensional functional CT. AJNR Am J Neuroradiol 1996;17:1861-69

3. Wittkamp G, Buerke B, Dziewas R, et al. Whole brain perfused blood volume CT: visualization of infarcted tissue compared to quantitative perfusion CT. Acad Radiol 2010;17:427-32

4. Latchaw RE, Alberts MJ, Lev MH, et al. Recommendations for imaging of acute ischemic stroke: a scientific statement from the American Heart Association. Stroke 2009;40:3646-78

S.P. Kloska

Department of Neuroradiology University of Erlangen-Nuremberg Erlangen, Germany and Department of Clinical Radiology University of Muenster Muenster, Germany

DOI 10.3174/ajnr.A2493 